\title{
Quantitative assessment of available probiotic products in community pharmacies in Benin City, Nigeria
}

\author{
Enosakhare Oloton*, Emmanuel Obaseki \\ Department of Pharmaceutical Microbiology and Biotechnology, Faculty of Pharmacy, University of Benin, Benin City, Nigeria
}

*For correspondence: Email: enosakhare.oloton@uniben.edu; Tel: +234-8036541222

Sent for review: 9 March 2020

Revised accepted: 15 June 2020

\begin{abstract}
Purpose: To assess four probiotic products available in community pharmacies in Benin City, Nigeria for accuracy of information on product labels with regard to the quantity and type of microorganisms, $\mathrm{pH}$ and bile tolerance, and antimicrobial activity.

Methods: Percent label compliance of products was determined, in addition to isolation, identification and enumeration of microorganisms. Determination of $\mathrm{pH}$ and bile tolerance was conducted using turbidity studies in MRS broth. Antimicrobial activity against Pseudomonas aeruginosa, Escherichia coli, Bacillus subtilis, Klebsiella pneumonia, Staphylococcus aureus and Candida albicans pathogens was investigated using agar overlay technique.

Results: In each product, there was $100 \%$ label compliance with regard to name of probiotic organism, storage condition, dose, expiration date, contact details and batch number. Three-quarters (75\%) of the probiotic products indicated product net quantity, National Agency for Food and Drug Administration and Control (NAFDAC) number, and microbial count; $50 \%$ of products indicated the excipients used, while only $25 \%$ of the products showed their indications. None of the products indicated strain designation. In species identification, Enterococcus faecium was absent in a multi-species product PB1, while PB3 contained Saccharomyces cerevisiae instead of Saccharomyces boulardii. Enumeration showed comparatively low quantities of probiotic organisms. Tolerance to $\mathrm{pH} 3$ and $\mathrm{pH} 7$, and bile levels of 0.3 and $2 \%$ were within acceptable range. The probiotic organisms demonstrated antimicrobial effect specifically against $P$. aeruginosa, E. coli, B. subtilis, K. pneumonia, S. aureus and C. albicans.

Conclusion: Antimicrobial effect and tolerance to $\mathrm{pH}$ and bile salts were consistent with acceptable properties of probiotics. However, there is need for total compliance with the indications, strain designation, excipients, and actual quantity of the individual probiotic organisms in the formulations.
\end{abstract}

Keywords: Probiotics, Strain designation, Agar overlay technique, Saccharomyces cerevisiae, Saccharomyces boulardii

\begin{abstract}
This is an Open Access article that uses a fund-ing model which does not charge readers or their institutions for access and distributed under the terms of the Creative Commons Attribution License (http://creativecommons.org/licenses/by/4.0) and the Budapest Open Access Initiative (http://www.budapestopenaccessinitiative.org/read), which permit unrestricted use, distribution, and reproduction in any medium, provided the original work is properly credited.
\end{abstract}

Tropical Journal of Pharmaceutical Research is indexed by Science Citation Index (SciSearch), Scopus, International Pharmaceutical Abstract, Chemical Abstracts, Embase, Index Copernicus, EBSCO, African Index Medicus, JournalSeek, Journal Citation Reports/Science Edition, Directory of Open Access Journals (DOAJ), African Journal Online, Bioline International, Open-J-Gate and Pharmacy Abstracts

\section{INTRODUCTION}

Probiotics are active microorganisms which, when prescribed in sufficient quantity, provide specific health benefit to the end users [1]. Some microorganisms such as Sporolactobacillus, Lactococcus, Propionibacterium, Escherichia coli and Leuconostoc mesenteroides [2,3] exhibit similar probiotic properties. These organisms have gained recognition because of their various 
health benefits such as prevention of diarrhea in children, modulation of immunity, alleviation of lactose intolerance, prevention of some forms of cancers, and lowering of serum cholesterol [4]. Moreover, it has been reported that some of these organisms prevent the occurrence of infectious diseases [5].

The nomenclature "probiotic" refers to supplements, foods and drugs for human and veterinary use [4]. Regulatory authorities require that the storage condition, expiration date, names of species, strain designations and their corresponding quantities be indicated on the label of each probiotic formulation [6]. It has been suggested that approximately $10^{8}$ to $10^{9}$ probiotic organisms should be prescribed daily for achievement of required health benefits [4].

Healthcare professionals in Nigeria seem to have limited awareness and knowledge of the use of probiotic products, notwithstanding their beneficial effects, which with time can be improved upon by pharmacists [7]. In specific terms, the pharmaceutical microbiologist has a crucial role of ensuring that the emerging awareness and knowledge of probiotic effects are used to match label claims with actual contents so as to ensure the achievement of effective pharmaceutical care in Nigeria.

The present study assessed four probiotic products available in community pharmacies in Benin City, Nigeria, with respect to the accuracy of information on product labels regarding the quantity and types of microorganisms, $\mathrm{pH}$ and bile tolerance, and antimicrobial effects.

\section{EXPERIMENTAL}

\section{Collection of samples of probiotic products}

Four available probiotic products (designated PB1, PB2, PB3 and PB4) were purchased from community pharmacies in Benin City, Edo state, Nigeria. The products were kept appropriately in a cool, dry environment, away from light for two days before the commencement of the study. Information such as name of probiotic organism (genus and species), strain designation, indication, batch number, product net quantity, dose and contact details were recorded. Then, the percentage compliance of the information on each product label was determined.

\section{Isolation and identification of probiotic microorganisms}

The content of a capsule or sachet of each product was dispersed in $9 \mathrm{~mL}$ of sterile phosphate buffered saline (PBS), $\mathrm{pH} 7.4$ and mixed thoroughly. Then, ten-fold serial dilutions were made in PBS, and appropriate dilutions of PB1-PB4 were plated onto appropriate agar media, and incubated under specific conditions. Dilutions of PB1 were plated on MRS agar (De Man Rogosa and Sharpe) adjusted with $0.05 \%$ (w/v) L-cysteine hydrochloride under anerophilic condition. The PB1 dilutions plated on MRS agar were also incubated under microaerophilic condition. Moreover, PB1 dilutions were plated on nutrient agar incubated under aerobic condition. The PB2 and PB3 dilutions were plated on Sabouraud Dextrose Agar (SDA) and incubated aerobically, while PB4 dilutions were plated on MRS agar and incubated under microaerophilic condition. The seeded agar plates were incubated at $37^{\circ} \mathrm{C}$ for $48 \mathrm{~h}$. Discrete colonies were isolated and labelled. Gram staining status, catalase status [8] and sugar fermentation tests were carried out using ribose, galactose, maltose, mannitol, sucrose, Dfructose, D-glucose and D-lactose. The probiotic microorganisms were identified based on bacterial sugar utilization as measured using Bergey's Manual of Determinative Bacteriology [9], and fungal utilization based on established methods [10]. The confirmed colonies were preserved for further studies.

\section{Enumeration of actual probiotic organisms on selective media}

The content of a capsule or sachet of each product was dispersed in $9 \mathrm{~mL}$ sterile phosphate buffered saline (PBS), $\mathrm{pH} 7.4$, and homogenized thoroughly. Then, ten-fold serial dilutions were prepared in PBS, and appropriate dilutions of PB1-PB4 were plated onto appropriate selective media and incubated under specific conditions. The PB1 dilutions were plated on MRS agar adjusted with $0.05 \% \quad(w / v) \quad$ L-cysteine hydrochloride under anerophilic condition; PB1 was plated on nutrient agar and incubated under aerobic condition; PB1 dilutions plated on MRS agar were also incubated under microaerophilic condition. The PB2 and PB3 dilutions were plated on Sabouraud Dextrose Agar (SDA) and incubated aerobically, while, PB4 dilutions were plated on MRS agar incubated under microaerophilic condition. The seeded agar plates were incubated at $37^{\circ} \mathrm{C}$ for $48 \mathrm{~h}$. Colonies were counted at the end of incubation, and the actual number of viable probiotic organisms in each product was expressed in colony-forming units (cfu) per $\mathrm{mL}$. The identified probiotic organisms were compared with those indicated on the product labels.

Trop J Pharm Res, July 2020; 19(7): 1512 


\section{Studies on $\mathrm{pH}$ tolerance of probiotic microorganisms}

The $\mathrm{pH}$ tolerance was determined by first culturing the isolates on appropriate broth (MRS broth and Nutrient broth) for $20 \mathrm{~h}$ at $37^{\circ} \mathrm{C}$. Then, $1 \times 10^{6} \mathrm{cfu} / \mathrm{mL}$ of each isolate was added to 10 $\mathrm{mL}$ of appropriate broth adjusted to $\mathrm{pH}$ values of 1.5 , 3.0 or 7.0 , using $0.1 \mathrm{M} \mathrm{HCl}$. The broth cultures were incubated for $24-48 \mathrm{~h}$ at $37^{\circ} \mathrm{C}$; microbial survival determined on turbidity [11].

\section{Studies on bile salt tolerance of probiotic microorganisms}

In the determination of bile salt tolerance, the isolates were first cultured on appropriate liquid media for $20 \mathrm{~h}$ at $37^{\circ} \mathrm{C}$. Specifically, $20 \mu \mathrm{L}$ of each isolate containing $1 \times 10^{6} \mathrm{cfu} / \mathrm{mL}$ was added to $10 \mathrm{~mL}$ of appropriate broth adjusted to bile salt levels of 0.3 and $2 \%$. The inoculated broths were incubated for $24-48 \mathrm{~h}$ at $37^{\circ} \mathrm{C}$, depending on the organism, and percentage survival was determined based on turbidity of the broth culture [11].

\section{Determination of antimicrobial effects probiotic organisms}

The antimicrobial effects of probiotic organisms against some test pathogens (Pseudomonas aeruginosa, Escherichia coli, Bacillus subtilis, Klebsiella pneumonia, Staphylococcus aureus and Candida albicans) were determined with soft agar overlay technique, with some modifications [12]. In this process, MRS agar plates containing confluent growths of the probiotic isolates in spots ranging from 4 - $5 \mathrm{~mm}$ were overspread with soft Muller-Hinton agar pre-inoculated with the test pathogens. The overlaid media were allowed to set and subsequently incubated at 37 ${ }^{\circ} \mathrm{C}$ for $24-48 \mathrm{~h}$. The diameter of zone of inhibition (DZI) was recorded as index of antimicrobial effect, while the absence of DZI was considered as lack of antimicrobial effect.

\section{Statistical analysis}

Statistical analysis was performed using GraphPad InStat version 3.10 program. Test for statistical significance were done using KruskalWallis non-parametric ANOVA, paired $t$-test and one-way analysis of variance (ANOVA). Differences were considered statistically significant at $p \leq 0.05$.

\section{RESULTS}

Each of the probiotic products indicated the contact details, expiration date, dose, batch number, storage instructions and names of the organisms up to species level. Seventy-five percent $(75 \%)$ of the products provided information with respect to NAFDAC approval number, product net quantity and microbial count. Fifty percent $(50 \%)$ of the investigated probiotic products indicated the excipients used in the preparations. However, only $25 \%$ of the products showed indications, while none of the products showed strain designation. These results are presented in Table 1.

Table 1: Content labels of probiotic products

\begin{tabular}{lc}
\hline Label content & Compliance (\%) \\
\hline Contact details & 100 \\
Expiration dates & 100 \\
Dose & 100 \\
NAFDAC number & 75 \\
Product net quantity & 75 \\
Excipients & 50 \\
Batch number & 100 \\
Storage instructions & 100 \\
Indications & 25 \\
Microbial count & 75 \\
Strain designation & - \\
Name-species & 100 \\
Name-genus & 100 \\
\hline
\end{tabular}

The identities of the selectively-isolated organisms and results of their respective Gram staining, catalase test and sugar utilization tests are shown in Table 2. The isolates obtained were labeled as $\mathrm{PB}^{\mathrm{a}}, \mathrm{PB}^{\mathrm{b}}{ }^{\mathrm{P}}, \mathrm{PB} 1^{\mathrm{c}}, \mathrm{PB} 2, \mathrm{~PB} 3$ and PB4. A careful review of the results showed that $\mathrm{PB} 1^{\mathrm{a}}$ was a Gram-positive and catalase-negative bacillus, with the utilizable sugars ribose, galactose, maltose, sucrose, D-fructose, Dglucose and D-lactose. Mannitol was the only non-utilizable sugar for $P B 1^{\mathrm{a}}$. Based on the selective medium used in its isolation $(0.05 \% \mathrm{w} / \mathrm{v}$ L-cysteine hydrochloride under anerophilic condition) and the results of the identification tests, $\mathrm{PB} 1^{\mathrm{a}}$ was identified as Bifidobacterium infantis.

The probiotic isolates $\mathrm{PB} 1^{\mathrm{c}}$ and PB4 were Grampositive and catalase-negative bacilli which utilized ribose, galactose, maltose, sucrose, Dfructose, D-glucose and D-lactose, but did not metabolize mannitol (non-utilizable sugar). These identification results, and the selective medium used for their isolation (MRS agar incubated in a microaerophilic condition) revealed the presence of Lactobacillus acidophilus.

Probiotic isolate PB2 was Gram-positive and catalase-positive cocci, with the utilizable sugars maltose, sucrose, D-fructose and D-glucose, and non-utilizable sugars ribose, galactose, mannitol and D-lactose. A positive identification of Saccharomyces boulardii was made, based on 
the selective medium used for isolating PB2 (SDA incubated in aerobic condition) and the results of the various identification tests.

The probiotic isolate PB3 showed a similar pattern as PB2 with respect to the selective medium used for its isolation, and identification tests, but differed specifically in the galactose utilization test: PB3 was able to utilize galactose, which is consistent with Saccharomyces cerevisiae.

It should be noted that Enteroccocus faecium which was indicated in the label of $\mathrm{PB} 1^{\mathrm{b}}$, was absent in the actual determination.

The quantity indicated on the label of PB1 did not tally with the relative quantity of the probiotic organism obtained. There was no indication of quantity of organisms on label of PB2, while the labels on PB3 and PB4 captured their respective quantities. Apart from $\mathrm{PB} 1^{\mathrm{b}}$, the actual quantities of all isolates ranged from $1.6 \times 10^{3}$ to $1.0 \times 10^{9}$ $\mathrm{cfu} / \mathrm{mL}$, as shown in Table 3.

The $\mathrm{pH}$ and bile salts tolerance are shown in Table 4. Bifidobacterium infantis, S. boulardii, $S$. cerevisiae, $L$. acidophilus (PB1) and $L$. acidophilus (PB4) showed growth in media of $\mathrm{pH}$ 3.0 and $\mathrm{pH} 7.0$, as well as in media containing 0.3 and $2.0 \%$ bile salts. However, there were no growths in a medium of $\mathrm{pH} 1.5$ (Table 4).

The antimicrobial effects of the probiotic organisms are shown in Table 5. The antimicrobial effect of $B$. infantis and the respective $D Z I$ ranges against the test pathogens were: 6 - $8 \mathrm{~mm}$ (C. albicans); 9-11mm (E. coli, K. pneumonia, and $P$. aeruginosa), and $13-15 \mathrm{~mm}$ (B. subtilis and $S$. aureus).

Table 2: Probiotic microorganisms in the probiotic products analyzed

\begin{tabular}{|c|c|c|c|c|c|c|}
\hline \multirow{2}{*}{$\begin{array}{l}\text { Identification } \\
\text { test }\end{array}$} & \multicolumn{6}{|c|}{ Probiotic isolate } \\
\hline & PB1 $^{a}$ & $P B 1^{b}$ & $P B 1^{C}$ & PB2 & PB3 & PB4 \\
\hline Gram staining & GPB & Absent & GPB & GPC & GPC & GPB \\
\hline Catalase & - & Absent & - & + & + & - \\
\hline Ribose & + & Absent & + & - & - & + \\
\hline Galactose & + & Absent & + & - & + & + \\
\hline Maltose & + & Absent & + & + & + & + \\
\hline D-fructose & + & Absent & + & + & + & + \\
\hline D-Glucose & + & Absent & + & + & + & + \\
\hline D-Lactose & + & Absent & + & - & - & + \\
\hline $\begin{array}{l}\text { Probiotic } \\
\text { organisms }\end{array}$ & $\begin{array}{c}B . \\
\text { infantis }\end{array}$ & - & L. acidophilus & S. boulardii & S. cerevisiae & L. acidophilus \\
\hline
\end{tabular}

GPC = Gram-positive cocci, GPB = Gram-positive bacilli

Table 3: Actual counts of probiotic organisms, in relation to contents shown on label content

\begin{tabular}{lllcc}
\hline $\begin{array}{l}\text { Probiotic } \\
\text { sample }\end{array}$ & \multicolumn{2}{c}{ Probiotic organism } & \multicolumn{2}{c}{ Quantity (cfu/mL) } \\
\cline { 2 - 5 } & Label & Actual & Label & Actual \\
\hline PB1 & B. infantis & B. infantis & $1.2 \times 10^{7 *}$ & - \\
PB1 PB1 & E. faecium & Absent & & $1.0 \times 10^{3}$ \\
PB1 & L. acidophilus & L. acidophilus & - & $1.0 \times 10^{9}$ \\
PB2 & S. boulardii & S. boulardii & $5.0 \times 10^{9}$ & $1.6 \times 10^{3}$ \\
PB3 & S. boulardii & S. cerevisiae & $1.0 \times 10^{8}$ & $1.0 \times 10^{5}$ \\
PB4 & L. acidophilus & L. acidophilus & \\
\hline
\end{tabular}

Bifidobacterium infantis = B.infantis, Saccharomyces cerevisiae $=S$. cerevisiae, Lactobacillus acidophilus $=L$. acidophilus, Saccharomyces boulardii $=$ S. boulardii, Enteroccocus faecium $=$ E. faecium, *Uncategorized

Table 4: Effect of $\mathrm{pH}$ and bile salts on growth of probiotic organisms

\begin{tabular}{|c|c|c|c|c|c|c|}
\hline \multirow{2}{*}{$\begin{array}{l}\text { Probiotic } \\
\text { organism }\end{array}$} & \multicolumn{3}{|c|}{ pH } & \multicolumn{3}{|c|}{ Bile salt (\%) } \\
\hline & 1.5 & 3.0 & 7.0 & 0 & 0.3 & 2.0 \\
\hline B. infantis & - & + & + & + & + & + \\
\hline L. acidophilus (PB1) & - & + & + & + & + & + \\
\hline S. boulardii & - & + & + & + & + & + \\
\hline S. cerevisiae & - & + & + & + & + & + \\
\hline L. acidophilus (PB4) & - & + & + & + & + & + \\
\hline
\end{tabular}


Table 5: Antimicrobial effects of the probiotic organisms

\begin{tabular}{|c|c|c|c|c|c|c|}
\hline \multirow[t]{2}{*}{ Probiotic organism } & \multicolumn{6}{|c|}{ Pathogenic organism } \\
\hline & $\begin{array}{c}B . \\
\text { subtilis }\end{array}$ & E. coli & K. pneumonia & P. aeruginosa & S. aureus & C. albicans \\
\hline B. infantis & +++ & ++ & ++ & ++ & +++ & + \\
\hline L. acidophilus (PB1) & +++ & ++ & + & + & ++ & + \\
\hline S. boulardii & ++ & ++ & ++ & + & ++ & + \\
\hline S. cerevisiae & + & + & + & + & ++ & + \\
\hline L. acidophilus (PB4) & ++ & + & + & + & ++ & + \\
\hline
\end{tabular}

$B$. subtilis =Bacillus subtilis, $E$. coli $=$ Escherichia coli, $K$. pneumonia =Klebsiella pneumonia, $P$. aeruginosa $=$ Pseudomonas aeruginosa, $S$. aureus $=$ Staphylococcus aureus, $=$ C. albicans $=$ Candida albicans. $(+=6-8$ $\mathrm{mm} ;++=9-11 \mathrm{~mm} ;+++=13-15 \mathrm{~mm}$ )

The antimicrobial effect of $L$. acidophilus (obtained from probiotic product PB1), and the respective $\mathrm{DZI}$ ranges against the test pathogens were: 6 - $8 \mathrm{~mm}$ (K. pneumonia, $P$. aeruginosa, and C. albicans); $9-11 \mathrm{~mm}$ (E. coli and $S$. aureus), and $13-15 \mathrm{~mm}$ (B. subtilis).

For $S$. boulardii the antimicrobial effect and ranges of $\mathrm{DZI}$ against the pathogens tested were: $6-8 \mathrm{~mm}$ ( $P$. aeruginosa and $C$. albicans) and $9-11 \mathrm{~mm}$ (B. subtilis, E. coli, K. pneumonia, and $S$. aureus). The antimicrobial effect of $S$. cerevisiae and ranges of DZI against the various pathogens tested were: 6 - $8 \mathrm{~mm}$ (B. subtilis, $E$. coli, $K$. pneumonia, $P$. aeruginosa and $C$. albicans); and $9-11 \mathrm{~mm}$ (S. aureus). For $L$. acidophilus (obtained from probiotic product PB4) the antimicrobial effect and ranges of DZI against test pathogens were: 6 - 8mm (E. coli, $K$. pneumonia, $P$. aeruginosa and $C$. albicans); and $9-11 \mathrm{~mm}$ (B. subtilis and $S$. aureus).

\section{DISCUSSION}

There was label compliance of $100 \%$ in the names of probiotic organisms which were shown in terms of genus and species, and also in storage condition, dose, expiration date, contact details and batch number, as expected for a probiotic drug. Results from label content study did not differ significantly from label indications. However, product net quantity, NAFDAC number, microbial count, excipient and indications were not fully stated. None of the probiotic products showed strain designation. The partial compliance and absence of strain designation seen in this study are consistent with results obtained in a similar study [13]. The incomplete labeling suggests that these products were not subjected to scrutiny by the relevant regulatory agencies in Nigeria. Since probiotic organisms are strain-specific, strain designation is necessary because it enables prescribers to match the probiotic organisms used in the products with their origins and health benefits.
There is a need for absolute compliance with the label contents of the probiotic formulations for the benefit of both the prescriber and the end users. In the isolation and identification of probiotic organisms, the indicated label contents of singlespecies products PB2 and PB4 i.e. S. boulardii and $L$. acidophilus, respectively, were consistent with their actual contents. The multiple-species product (PB1) ought to contain three probiotic organisms labelled $\mathrm{PB} 1^{\mathrm{a}}, \mathrm{PB} 1^{\mathrm{b}}$ and $\mathrm{PB} 1^{\mathrm{c}}$, based on the label. The labels of $\mathrm{PB} 1^{\mathrm{a}}$ and $\mathrm{PB} 1^{\mathrm{c}}$ probiotic organisms indicated contents of $B$ infantis and L.acidophilus which were also consistent with the actual contents, while $E$. faecium which was stated on the label of $\mathrm{PB} 1^{\mathrm{b}}$ was absent in the actual determination. Another single-species product PB3 actually contained S. cerevisiae instead of $S$. boulardii indicated on the label. The discrepancies between label claims and actual contents seen in this study are similar to findings in a related work [14]. These negative features may have resulted from omissions or losses during technological processing of the microorganisms, and they are likely to affect the overall synergistic and species-specific effects of the probiotic organisms. The absence of a particular strain from PB1 and the mislabeling in PB3 are not in conformity with the Food and Agriculture Organization (FAO) and World Health Organization (WHO) recommendations on products containing probiotics. Comprehensive review and strict adherence to standard operating procedures (SOPs) should be used to minimize the observed errors.

In the enumeration test, the label content for PB1 did not categorize the different probiotic organisms. This product actually contained low concentration of $\mathrm{PB}^{\mathrm{a}}(B$. infantis) and adequate concentration of $\mathrm{PB}^{\mathrm{C}}$ ( $L$. acidophilus). The quantity of probiotic organisms (S. boulardii) was not shown in label contents of PB2 with respect to adequate/actual concentration, while PB3 and PB4 actually contained lower concentrations than were stated in their respective labels, although 
the differences were not statistically significant. Several studies which evaluated the quality of probiotics have shown similar enumeration patterns [15]. Poor storage conditions of probiotic products in community pharmacies are likely to contribute to the low concentrations of organisms observed among the probiotic products analyzed.

All the probiotic organisms isolated from products PB1, PB2, PB3 and PB4 exhibited tolerance to $\mathrm{pH} 3.0$ and $\mathrm{pH} 7.0$, but they did not tolerate $\mathrm{pH}$ 1.5. A good source of probiotics should survive in a medium of $\mathrm{pH} 3.0$, which is consistent with what was observed in all the probiotic organisms isolated from the probiotic products [16]. This implies that all the probiotic organisms can withstand the denaturing effect of gastric acid during transition from the stomach to the duodenum. Moreover, the probiotic isolates showed tolerance to bile salts at levels of 0.3 and $2 \%$, which is within and above the $0.3 \%$ bile tolerance recommended for the selection or identification of tolerance of probiotic organisms for human use [17]. This demonstrates the ability of the probiotic organisms to resist the cell membrane-solubilizing effect of bile as they move through the duodenum to the distal ileum and colon. The tolerance of the organisms to $\mathrm{pH}$ and varying concentrations of bile salts were within acceptable ranges for the gastrointestinal viability of each of the studied probiotic products.

The individual probiotic species isolated from the pharmaceutical products demonstrated growthinhibitory properties as shown by their significant antimicrobial effects against $P$. aeruginosa, $E$. coli, B. subtilis, K. pneumonia, $S$. aureus and $C$. albicans. The observed antimicrobial properties of the isolates are consistent with results obtained in a similar investigation [18]. The lower $\mathrm{DZI}$ seen for $C$. albicans may have resulted from the morphological differences ( $C$. albicans is a fungus), when compared with other test organisms (bacteria) used in this determination. The low DZI values are consistent with the beneficial antimicrobial effects of probiotic organisms in the formulations.

\section{CONCLUSION}

Four products PB1, PB2, PB3 and PB4 have been analyzed in this study. The results reveal some degree of non-compliance of the products with regulatory requirements. There is an urgent need for compliance with extant regulatory specifications for probiotic products. The findings in this study point to the need for stricter regulation of the quality of probiotic products by the appropriate regulatory agencies.

\section{DECLARATIONS}

\section{Acknowledgement}

The authors express their sincere appreciation to the entire staff of the Department of Pharmaceutical Microbiology and Biotechnology, Faculty of Pharmacy, University of Benin, for providing the facilities that enabled them to carry out this study.

\section{Conflict of interest}

The authors declare that no conflict of interest is associated with this work.

\section{Contributions of authors}

We declare that this work was done by the authors named in this article, and all liabilities pertaining to claims relating to the content of this article will be borne by the authors. Oloton E. and Obaseki E. contributed to the study conceptualization and design, data acquisition, manuscript preparation, and critical revision of the manuscript. All the authors contributed significantly to the study, and they all agree with the contents of the manuscript.

\section{Open Access}

This is an Open Access article that uses a funding model which does not charge readers or their institutions for access and distributed under the terms of the Creative Commons Attribution License (http://creativecommons.org/licenses/by/ 4.0) and the Budapest Open Access Initiative (http://www.budapestopenaccessinitiative.org/rea d), which permit unrestricted use, distribution, and reproduction in any medium, provided the original work is properly credited.

\section{REFERENCES}

1. FAO/WHO. "Guidelines for the evaluation of probiotics in food," Report of a Joint FAO/WHO Working Group on Drafting Guidelines for the Evaluation of Probiotics in Food. 2002: 7-8.

2. Ayichew $T$, Belete A, Alebachew $T$, Tsehaye $H$, Berhanu $H$, Minwuyelet A. Bacterial Probiotics, their Importance and Limitations: a Review. J Nutr Health Sci 2017; 4(2): 202.

3. Kechagia M, Basoulis D, Konstantopoulou S, Dimitriadi D, Gyftopoulou K, Skarmoutsou N, Fakiri EM. "Health benefits of probiotics: A Review". ISRN Nutr 2013:1-7.

4. Kailasapathy K, Chin J. Survival and therapeutic potential of probiotic organisms with reference to Lactobacillus acidophilus and Bifidobacterium spp.

Trop J Pharm Res, July 2020; 19(7): 1516 
5. Hemaiswarya S, Raja R, Ravikumar R, Carvalho IS. Mechanism of Action of probiotics Braz Arch Biol Technol 2013; 56: 113-119.

6. Sanders ME. How Do We Know When Something Called "Probiotic" Is Really a Probiotic? A Guideline for Consumers and Health Care Professionals. Functional Food Reviews 2009; 1: 3-12.

7. Amarauche CO. Assessing the Awareness and Knowledge on the Use of Probiotics by Healthcare Professionals in Nigeria. J Young Pharm 2016; 8(1): 5355.

8. Cheesbrough M. District Laboratory Practice in Tropical Countries. 2nd Edn., Cambridge University Press, Cambridge, UK; 2006 (Pt 2): 35-70.

9. Holt JG, Krieg NR, Peter $H$, Sneath A, Williams ST. Bergeys Manual of Determinative Bacteriology. William and Wilkins Baltimore. 1994; 787 p

10. Łukaszewicz M. Saccharomyces cerevisiae var. boulardii - Probiotic Yeast. (Ed.) InTech 2012:388.

11. Gore S, Paul A, Bhagwat Y. Comparative evaluation of commercially available probiotics products. Int J Curr Pharm Res 2016; 9(2): 26-30.

12. Ran C, Carrias A, Williams MA, Capps N, Dan BCT, Newton JC, Kloepper JW, Ooi EL, Browdy CL, Terhune
JS, et al. Identification of Bacillus Strains for Biological Control of Catfish Pathogens. PLoS ONE 2012; 7(9) 2.

13. Sehgal S, Dhewa T, Bansal N, Shashank A, Sharma N, Thakur M, Himanshi AS, Mehta S, Anil PR, Jha A. Evaluation of labelling practices of probiotic products commercially available in Delhi Market. Du JURI 2015; 1(1): 219-221.

14. Weese JS. Evaluation of deficiencies in Labeling of commercial probiotics. Can Vet 2003; 44: 982-983.

15. Fredua-Agyeman M, Parab S, Gaisford S. Evaluation of commercial Probiotic products. Br J Pharm 2016; 1:8788.

16. Fernandez MF, Boris S, Barbes C. Probiotic properties of human lactobacilli strains to be used in the gastrointestinal tract. J Appl Microbiol 2003; 94: 449455.

17. Goldin, Berry R. Gorbach, Sherwood L. Probiotics for humans. In: Fuller, R. (Ed.), Probiotics, the Scientific Basis. Chapman \& Hall, London; 1992. p. 355-376.

18. Sayeed MA, Hossen A, Saha R, Jakaria M. Antimicrobial activities of isolated probiotics and their metabolites against some pathogenic microorganisms. IIUC Studies 2017; 14(1): 25-27. 